\title{
Spinocerebellar ataxia type 17
}

INSERM

\section{Source}

INSERM. (1999). Orphanet: an online rare disease and orphan drug data base.

Spinocerebellar ataxia type 17. ORPHA:98759

Spinocerebellar ataxia type 17 (SCA17) is a rare subtype of type I autosomal dominant cerebellar ataxia (ADCA type l; see this term). It is characterized by a variable clinical picture which can include dementia, psychiatric disorders, parkinsonism, dystonia, chorea, spasticity, and epilepsy. 